\title{
Role of Graphs in Blending Physical and Mathematical Meaning of Partial Derivatives in the Context of the Heat Equation
}

\author{
Sofie Van den Eynde ${ }^{1,2}$ (D) Martin Goedhart ${ }^{1} \cdot$ Johan Deprez $^{3} \cdot$ Mieke De Cock $^{2}$
}

Received: 17 April 2020 / Accepted: 6 November 2021

(c) Ministry of Science and Technology, Taiwan 2021

\begin{abstract}
From literature, we know that making the connections between mathematics and physics is not trivial for most students, even at the advanced level. In the specific context of partial derivatives in thermodynamics, research suggests that making explicit connections between the mathematics and the physics is necessary to foster student understanding. In this paper, we investigate how graphical reasoning can help undergraduate students in making connections between the partial derivatives of temperature with respect to position and to time and their respective physical meaning in the context of one-dimensional systems modeled by the heat equation. We conducted task-based, think aloud interviews with four pairs of undergraduate students majoring in physics or mathematics and use dynamic conceptual blending diagrams to analyze their reasoning processes and the role of the graphs therein. The results in this paper indicate that stimulating graphical reasoning is a promising way to promote the blending of mathematical and physical knowledge. Specifically, our data shows that constructing graphs can be a catalyst which helps students to give physical meaning to the partial derivatives $\partial T / \partial t$ and/or $\partial T / \partial x$. Therefore, in teaching/learning activities, actively promoting graph construction and reasoning based on those graphs offers perspectives to support the blending of mathematics and physics.
\end{abstract}

Keywords Conceptual blending $\cdot$ Graphs $\cdot$ Undergraduate education · Partial derivatives

Sofie Van den Eynde

s.van.den.eynde@rug.nl

Mieke De Cock

mieke.decock@kuleuven.be

1 Institute for Science Education and Communication, Faculty of Science and Engineering, University of Groningen, Groningen, the Netherlands

2 Department of Physics and Astronomy \& LESEC, KU Leuven, Leuven, Belgium

3 Department of Mathematics \& LESEC, KU Leuven, Leuven, Belgium

Published online: 10 January 2022 


\section{Introduction}

Advanced physical concepts are expressed in terms of mathematical structures. Therefore, proficiency in mathematics is required to understand physical phenomena, and being able to combine the different disciplines is a prerequisite to become more proficient in physics (Redish \& Kuo, 2015). Using mathematics in physics requires more than the straightforward application of rules and mathematical procedures. Mathematics does not only have a technical role, but also a structural one (Uhden et al., 2012). This implies that mathematics is deeply interwoven with physics: they are often even inseparable. Studying students' use and understanding of the mathematics used in physics is an important and central research theme in physics education research (PER) (e.g. Hu \& Rebello, 2013; Johansson, 2016; Karam, 2015; Niss, 2017; Planinic et al., 2012; B. P. Schermerhorn \& Thompson, 2019; Wilcox et al., 2013). Parsing students' mathematical and physical understanding has proven challenging as the use of mathematics in physics is more than just the sum of both parts. In this study, we investigate how graphs can play a role in connecting mathematical and physical knowledge. More specifically, we use the conceptual blending framework (Fauconnier \& Turner, 2003) to analyze the role of graphs in student reasoning when they describe physical systems that can be modeled by the heat equation, a partial differential equation.

We start by briefly reviewing the heat equation as a partial differential equation and the way it describes heat transfer in a system. We then give an overview of the relevant literature on partial derivatives in thermodynamics and the role of graphical reasoning in connecting mathematical and physical knowledge. Then, we introduce the conceptual blending framework, which is the theoretical framework that guides this study. Next, we discuss the methodology, both the data collection and data analysis. In the "Results" section, we discuss five case studies. We end with a discussion and an overview of limitations and suggestions for future research.

\section{The Heat Equation as a Context}

The heat equation is one of the "standard" partial differential equations treated in the undergraduate program of physics and mathematics majors. In this study, we provided students with physical situations that can be described by the one-dimensional version of the heat equation. This partial differential equation models the evolution of the temperature at position $x$ and time $t$ in a one-dimensional system of length $L$ :

$$
\frac{\partial T}{\partial t}(x, t)=\alpha \frac{\partial^{2} T}{\partial x^{2}}(x, t)
$$

for $0<x<L$ and $0<t<\infty$. In this equation, $\alpha$ is the thermal diffusivity $\left[\mathrm{m}^{2} / \mathrm{s}\right]$, a measure of the rate at which heat can spread in a specific medium.

The equation relates the quantities $\partial T / \partial t$, the rate of change in temperature with respect to time, and $\partial^{2} T / \partial x^{2}$, the concavity of the temperature distribution $T(x, t)$, 
which essentially compares the temperature at one point to the temperature at neighboring points (Farlow, 1993).

To fully describe the system, the initial temperature distribution $T(x, t=0)$ and the conditions describing what happens at the boundaries of the system are needed. Boundary conditions refer to the conditions physical quantities must satisfy at the boundaries of the system at all times. A first type of boundary condition that we use in this paper has the form $T\left(x_{b}, t\right)=c$ with $c$ being a constant specifying the value of the temperature at the boundary $=x_{b}$. A second type of boundary condition has the form $\partial T / \partial x\left(x_{b}, t\right)=c$ with $c$ being a constant, giving information about the heat flowing through the boundary $x=x_{b}$. This can be explained by the law of thermal conduction, also known as Fourier's law, which states that the heat that flows through a unit area per unit of time is proportional to the negative gradient of the temperature. In the one-dimensional differential form, this law can be written as follows:

$$
q(x, t)=-k \partial T / \partial x(x, t)
$$

where $q(x, t)$ is the amount of heat that flows through a unit area per unit of time $\left[\mathrm{Wm}^{-2}\right] . k$ is the thermal conductivity $\left[\mathrm{Wm}^{-1} \mathrm{~K}^{-1}\right]$, which is a measure of how well the material conducts heat, and is related to the thermal diffusivity $\alpha$ as $\alpha=k / \rho c_{p}$ with $\rho$ the density $\left[\mathrm{kgm}^{-3}\right]$ and $c_{p}$ the specific heat capacity $\left[\mathrm{Jkg}^{-1} \mathrm{~K}^{-1}\right]$ (Farlow, 1993). This shows that the derivative of temperature with respect to position, which expresses the local temperature difference, is a measure for the heat transferred. Fourier's law states that if $\partial T / \partial x\left(x_{0}, t\right)<0$, then the flow of heat through point $x_{0}$ will be from left to right (given the $x$-axis is oriented to the right) because heat always flows from high to low temperatures; similarly if $\partial T / \partial x\left(x_{0}, t\right)>0$, then the flow will be from right to left.

\section{Mathematics and Physics in Thermodynamics}

From prior work, we know that students have difficulties formulating the relation between the derivative $\partial T / \partial x$ of temperature with respect to position, and heat flow (Authors, 2021). The much researched temperature-heat confusion (e.g., Clough \& Driver, 1986; Goedhart \& Kaper, 2003; Kesidou \& Duit, 1993; Linn \& Songer, 1991), i.e., the difficulty to distinguish between temperature and heat, might play a role here, because students have to distinguish clearly between both concepts and understand what the derivative of temperature with respect to position means in order to draw conclusions about the heat flow. Furthermore, several studies have investigated student understanding of partial derivatives in the context of upper-division thermodynamics. Becker and Towns (2012) confirm what we know from many other studies: rigorous understanding of mathematical ideas does not always ensure that students will be able to successfully relate mathematical knowledge to science content. To make sense of the partial derivatives, the students have to reinterpret the mathematics in new contexts, like thermodynamics. Moreover, Becker and Towns state that students may need significant guidance from instructors throughout this process. Thompson et al. (2006) state that students have a largely algorithmic, rather 
than conceptual understanding of partial derivatives. Their results suggest that making the connections between mathematics and physics, even at the advanced level, is not trivial for most students. In addition, failure to make these connections in both directions may prevent a full understanding of the relevant physical phenomena, at least in thermodynamics. In follow-up research, Thompson et al. (2012) investigated ways to scaffold these connections between mathematics and physics. In the activities they designed, they explore two possible ways to connect the partial derivatives to physical meaning. The first way is by letting students describe an experiment to measure what the partial derivative is representing. Secondly, students are asked to link partial derivatives with their graphical representation in three-dimensional plots. Their results show that by the end of the set of activities, most students of the class recognize the connections between the mathematics and the physics easily. This success is attributed to the deliberate effort to make connections between mathematics and physics explicit, including making students aware of differences in disciplinary notations and conventions. Roundy et al. (2014) built on this effort to make explicit connections between mathematics and physics by extending the work of Thompson and colleagues with more concrete activities, to be used at different times in a thermodynamics course, where students have to come up with a physical experiment when given a partial derivative. In all designed activities, there is a lot of attention to the importance of physical meaning and relevance.

\section{Graphical Reasoning in Connecting Mathematics and Physics}

Like the research discussed in the previous paragraph, we are also looking for ways to help students in connecting mathematics and physics. In our data, we observed that some students spontaneously construct graphs when trying to give physical meaning to the partial derivatives $\partial T / \partial x$ and $\partial T / \partial t$. Moreover, we observed that sketching graphs and reasoning based on these graphs (e.g., using slope) often helped the students to take a next step in their reasoning process.

A vast amount of research studied students' understanding and construction of graphs. Reviews have been published by Leinhardt et al. (1990) and Glazer (2011) with a focus on mathematics education and science education, respectively. Most studies concern secondary students' graph comprehension, highlighting the role of students' pre-knowledge and misconceptions (e.g., Leinhardt et al., 1990; McDermott et al., 1987), and the role of visual factors (e.g., Arcavi, 2003; Shah et al., 1999). Most research has been done in mathematics classes with graphs representing functions (Leinhardt et al., 1990). Studies about graph interpretation and construction outside the mathematics area are relatively rare (Glazer, 2011). Beichner (1994) developed a test to determine students' interpretations of kinematics graphs, modified by Zavala and colleagues (Zavala et al., 2017). These studies identified frequently occurring misconceptions among high school and college students. It appears that knowledge of the context is crucial in graph interpretation: Roth and Bowen (2003) show that even expert scientists had difficulties when interpreting graphs if they were not familiar with the kind of data or representation modes. 
In our context, we aim at the translation from a mathematical equation (representing a set of functions satisfying a certain mathematical property) to the description of a physical situation, and vice versa. Some studies on students' understanding of symbolic and visual representations, especially at undergraduate level, are relevant. Zazkis (2013) developed the VAP-model to analyze students' visual (V), analytical (A), and physical (P) modes of reasoning, and the transitions between reasoning with graphs, functions, and physical concepts. In an example, the author shows the transitions a student made when solving a derivative task, indicating that in a specific context, students are able to make transitions between different modes of reasoning. A study by Haciomeroglu et al. (2010) showed that students prefer different types of reasoning when solving calculus tasks: some students used an analytical approach, relying on symbolic representations, while other students preferred a visual approach with the aid of graphs. The aforementioned research by Thompson et al. (2012) mentioned that fostering the connections between the symbolic representation and the graphical interpretation of a partial derivative helped students in recognizing the connections between mathematics and physics. In chemistry, Rodriguez et al. (2018) investigated the role of graphs and equations in the blending of chemistry and mathematics. They interviewed undergraduate students as they worked through chemical kinetics problems. Their findings suggest that, in order for students to integrate chemistry and mathematics in a way that supports their understanding of the chemical processes, they must be able to reason using symbolic (Sherin, 2001) and graphical forms (Rodriguez et al., 2019, 2020), i.e., intuitive mathematical ideas the students associate with equations and graphs, respectively. Examples of graphical forms are steepness as rate, and straight means constant. Rodriguez et al. observed that often when students used these graphical forms, it aided their understanding of the chemical phenomena being modeled, leading them to discuss the mathematical narrative represented in the graph. They also found that graphical forms were mostly used by students who were making connections between chemistry and mathematics actively and regularly. Therefore, incorporating graphs in the reasoning could be a sign of a successful combination of mathematics with the other discipline because successful interpretation of a graph involves a combination of mathematical expertise and discipline-specific content to reason about the relationship between the variables and to describe the phenomena represented (Rodriguez et al., 2019, 2020).

\section{The Conceptual Blending Framework as a Lens}

Many authors have studied the relation between mathematics and physics using different frameworks (e.g., Arnon et al., 2014; Eichenlaub \& Redish, 2019; Kaiser et al., 2011; Karam, 2015; Pospiech et al., 2019; Uhden et al., 2012; Wilcox et al., 2013). Among them, several authors have adopted the conceptual blending perspective (Fauconnier \& Turner, 2003) to describe student understanding at the mathematics and physics interface (e.g., Bing \& Redish, 2007; Bollen et al., 2016; Hu \& Rebello, 2013). Conceptual blending was originally introduced by Fauconnier and Turner (1998) in linguistics and has been used in various contexts to explore 
human information integration (e.g., Bain et al., 2018; Gregorcic \& Haglund, 2021; Zandieh et al., 2014). It provides a way to describe individuals' knowledge construction through the integration or blending of ideas from different mental spaces. A mental space is comprised of conceptual packets or knowledge elements that tend to be activated together, and has an organizing frame that specifies the relationships, or connections between the elements. The blended space is constructed through selective projection from the input spaces. When used in the context of the math-physics interplay, the conceptual blending framework provides a language to discuss how students draw from physical and mathematical knowledge in their reasoning. Huynh and Sayre (2019) argue that "the effective use of mathematics in making sense of the physical world involves blending reciprocally between mathematics and physics contexts rather than just applying mathematics to physics."

In this paper, we take a dynamic conceptual blending perspective. In prior work (Authors, 2020), we presented dynamic blending diagrams (DBDs) as an analysis method that builds on earlier blending work in PER and MER (Bing \& Redish, 2007; Bollen et al., 2016; Gerson \& Walter, 2008; Hu \& Rebello, 2013; B. Schermerhorn, 2018). It incorporates the dynamic aspect of student reasoning and explicitly visualizes how students switch between mathematics, physics, and blended elements throughout their reasoning process. More details about the DBDs are discussed in the "Methodology" section. Using a dynamic blending perspective, we study in this paper how graphs and reasoning based on those graphs can help students in connecting the physical and mathematical meaning of the partial derivatives $\partial T / \partial x$ and $\partial T / \partial t$. Accordingly, our research question is as follows:

What is the role of graphs and graphical reasoning in the blending of physics and mathematics by undergraduate students in the context of the heat equation?

\section{Methodology}

\section{Participants and Context}

We conducted task-based, think-aloud interviews with four pairs of students to investigate their understanding of boundary conditions in the context of the heat equation. The participating students were second year physics and mathematics undergraduate students studying at University $\mathrm{X}$ and they participated in our study voluntarily. A few weeks before the interview, they finished a course on ordinary and partial differential equations, which entailed a chapter in which the heat equation was discussed, i.e., the derivation of the heat equation, boundary conditions, physical systems described by this equation, and the technique of separation of variables to solve the equation.

In our previous study (Authors, 2021), we observed that students interviewed individually had many difficulties reasoning about boundary conditions for the diffusion equation and were often unable to solve an interview task where they had to mathematically formulate boundary conditions for a physical situation. By letting students work in pairs, we hoped that they would be able to help each other. 
1. A metal rod is 1 meter long. Assume that heat can only flow along the rod. The initial temperature distribution in the rod is given in the following graph:

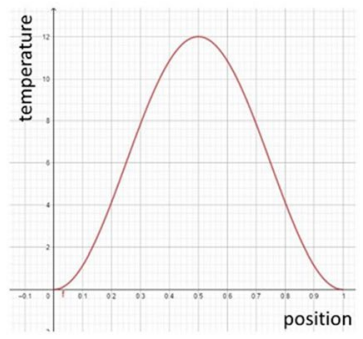

Both ends of the rod are isolated, which means that no heat can flow in or out of the rod.

A. Describe the situation the graph is referring to.

B. What is going to happen after some time? Sketch the temperature distribution in the rod at different times and explain important features of your graphs.

C. Explain what happens at the boundaries. How do you express that mathematically?

Explain your reasoning.

Fig. 1 Interview task 1 with its three subquestions

2. This PDE in combination with an initial condition (IC) and boundary conditions (BC) describes a specific physical situation and its evolution.

$$
\begin{gathered}
\frac{\partial u}{\partial t}(x, t)=\alpha \frac{\partial^{2} u}{\partial x^{2}}(x, t) \\
\text { IC: } u(x, 0)=f(x)
\end{gathered}
$$

with $f(x)$ an unspecified, continuous function on $[0,1]$

BCs: depending on question (A, B, C or D)

I. Describe a physical situation that matches these $\mathrm{BC}$ and describe it in a couple of sentences.

II. Discuss what the $\mathrm{BC}$ mean for the evolution of the values of temperature and heat flow.

III. Discuss what the $\mathrm{BC}$ mean for the values of temperature and heat flow after a very long time.

Fig. 2 Interview task 2 with its three subquestions. Task 2 is asked in four versions, each with a different set of boundary conditions (marked as A, B, C, and D). These are specified in Fig. 3

Moreover, in pairs, students would naturally question each other, forcing them to be more explicit about their reasoning process.

\section{Interview Content}

Figures 1, 2, and 3 show the interview tasks. In the task formulation, we use the notations from the course. This explains why we use $u(x, t)$ to describe the temperature in function of position and time, instead of the more common $T(x, t)$. The 
Fig. 3 The four sets of boundary conditions in interview task 2
A. BCs: $u(0, t)=0$
and $u(1, t)=0$
C. $\mathrm{BCs}: \frac{\partial u}{\partial x}(0, t)=0$
and $\frac{\partial u}{\partial x}(1, t)=0$
B. $\quad$ BCs: $u(0, t)=0$
and $u(10, t)=50$
D. $\quad$ BCs: $\frac{\partial u}{\partial x}(0, t)=-2$ and $u(1, t)=4$

interview tasks were not explicitly designed to study the use of graphs, but rather to investigate how students combine mathematical and physical concepts in the context of one-dimensional systems described by the heat equation. The focus on graphs only appeared after preliminary analysis of the data. All students answered all tasks. In task 1, we explicitly ask students to sketch graphs. Students should realize that the graph represents a situation in which no heat is transferred across the boundaries $(\partial u / \partial x=0)$. The temperatures near the boundaries will increase and near the middle of the rod will decrease until temperatures are equal at all positions. In our analysis of the answers to this task, we focus on the role of these constructed graphs on the reasoning. In task 2 (we asked four different versions $2 \mathrm{~A}, 2 \mathrm{~B}, 2 \mathrm{C}$, and 2D), we ask students to interpret boundary conditions, representing either a constant temperature $(u=$ constant), an isolated system (no heat flow, $\partial u / \partial x=0$ ), or a constant heat flow $(\partial u / \partial x=$ constant $)$ at the boundaries, and predict the evolution of the temperature and heat flow in the system. We do not prompt students to use graphs, but, in the analysis, we saw that some students spontaneously constructed graphs as part of their reasoning process.

\section{Interview Protocol}

Each interview lasted approximately one hour, during which the participants were encouraged to explicitly discuss and think aloud as they worked through the tasks. The interviewer and first author of the paper was not involved in the course. We used a smart-pen, which audio-recorded the conversations and kept track of the students' notes, drawings, and calculations.

At the start of the interview, the interviewer informed the students about the subject and purpose of the study. She also emphasized that she would not give feedback about the correctness of responses during the interview. In case students did not understand the question, the interviewer provided clarity. After this introduction, the students signed an informed consent form. Generally, the interviewer did not interfere, except for prompts to think aloud and requests for further explanation of what the students did and why. After the interview, the students had the chance to discuss their answers and the aim of the research project in an informal way.

\section{Data Selection and Analysis}

As mentioned in the introduction, we observed during these interviews that constructing graphs triggered students in their reasoning. In order to investigate the 


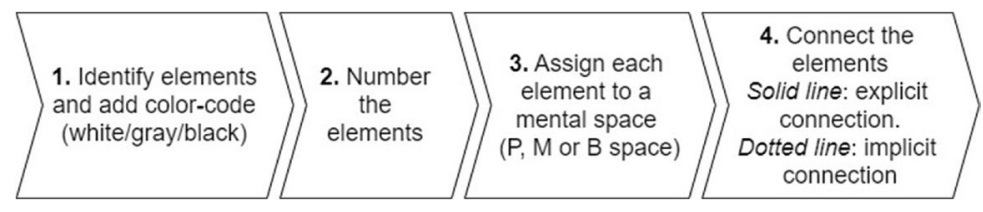

Fig. 4 Schematic overview of the four steps in constructing a dynamic blending diagram

Table 1 Color codes used in the dynamic blending diagrams

\begin{tabular}{ll}
\hline Color code & Meaning \\
\hline $\begin{array}{l}\text { Gray box } \\
\text { Black box }\end{array}$ & $\begin{array}{l}\text { Elements from the problem statement } \\
\text { Elements that are physically or math- } \\
\text { ematically incorrect in the context of the } \\
\text { question }\end{array}$ \\
White box & All other elements \\
\hline
\end{tabular}

effects of this graphical reasoning, we selected these parts of the data where graphical reasoning played a role, resulting in five cases consisting of answers to different interview tasks from three student pairs.

In prior work (), we developed the method of constructing dynamic blending diagrams (DBDs) with the aim of refining the conceptual blending framework as used in PER and MER to not only capture the product but also the process of student reasoning when combining physics and mathematics. The DBDs allow us to study in detail when and how students bring graphs into their reasoning. We give a review of the method to construct a DBDs, but we refer to for more extensive information and examples. Starting from a transcript, the DBDs is constructed in four steps as schematically shown in Fig. 4.

In step 1 in Fig. 4, an element is defined as a step, idea, concept, or phrase in the reasoning. By including the literal elements from the transcript, we minimize bias and interpretation. Most elements are utterances of the students, but, when actively used by the students, the elements from the task are included in the DBDs. A color code enables the reader to distinguish between the different types of elements in the DBDs (Table 1).

In this study, we focus on the role of graphs in student reasoning. Therefore, we select only the parts of the transcript where graphs play a role. In the analysis, we considered the complete transcript and all elements therein. In this paper however, for readability, we only represent the necessary elements to visualize the main line of reasoning.

As a second step in the construction of a DBDs, we visualize the reasoning process by numbering the elements in order of appearance in the transcript. In the third step, we assign each element to a mental space. We identify three spaces in our analysis: a physics space, a mathematics space, and a blended space. Table 2 shows the characterization of the different spaces. The role of graphs is of special interest in this project. Graphs are identified as elements in the mathematical space if these only refer to mathematical concepts, like function, derivative, and slope. If students 
Table 2 Characterization of the different mental spaces

\begin{tabular}{ll}
\hline Space & Definition \\
\hline Mathematics & $\begin{array}{c}\text { The mathematics space (M space) contains all elements related to mathematical knowl- } \\
\text { edge, without any new physical input (i.e., using only physical input that has already } \\
\text { been mentioned before in the reasoning process). This broadly entails mathematical } \\
\text { concepts, functions, and operations such as derivatives. This also entails graphs and } \\
\text { equations when they are used in an abstract, mathematical way without referring } \\
\text { explicitly to a particular physical situation } \\
\text { The physics space (P space) contains all elements related to physics knowledge, without } \\
\text { any new mathematical input. There can be a variety of levels: using physics concepts; } \\
\text { describing a physical system/process/relation in words; using experimentally known } \\
\text { facts or relations between physical quantities; statements about the nature or state of } \\
\text { the physical system } \\
\text { The blended space (B space) contains elements that combine mathematics and physics } \\
\text { ideas. Equations and graphs are categorized as part of the blended space when they } \\
\text { connect physical and mathematical meaning } \\
\text { This means that the boundary conditions given in the problem statement will be placed } \\
\text { here if students give a physical interpretation of these equations in their answer }\end{array}$ \\
Blended &
\end{tabular}

construct a graph and refer to both mathematical and physical concepts, like temperature, heat flow, or heat transfer, we categorize it as an element in the blended space.

In the last step, we relate the numbered elements in the DBDs to visualize the connections in the students' reasoning. We distinguish between explicit and implicit connections. When students explicitly connect elements in their reasoning, we connect the elements with a solid line. We use a dotted line if, based on the context in the transcript, we assume that the students connect the elements, even if they do not explicitly mention the connection. Students sometimes notice a contradiction in their reasoning. We represent this in the diagram by a solid line with arrows on both ends. By not only representing the mental spaces but also the connections made by the students using time stamps, we visualize the blending process in student reasoning.

\section{Reliability}

For reasons of reliability, two authors independently constructed a DBDs starting from transcriptions from three different, randomly selected, segments of the interviews that were not analyzed before (we will refer to them as R1, R2, and R3). We discuss the reliability on four levels: the selection of elements, the categorization of elements, the number of connections, and the type of connection. The percentages of agreement for every level are shown in Table 3 .

As part of the first stage, selection of elements, we listed the identified elements for both authors and checked for agreement. We calculated the percentage of agreement by dividing the number of agreeing elements by the total number of elements identified. For the second stage, we checked the placement of each element in the M, P, or blended space. Because the agreement in selection was high, we limited our further analysis to the common elements. We calculated a percentage of agreement by dividing the number of agreeing categorizations by the number of 
Table 3 Percentages of agreement between two authors that coded three pieces of data independently (R1, R2, and R3). For each piece of data, we add the number of identified elements between brackets (\# el.). Reliability is discussed on four levels: the selection of elements, the categorization of elements, the number of connections, and the type of connection

\begin{tabular}{lllll}
\hline & Selection & Categorization & Connections & Type \\
\hline R1 (11 elements) & 90.9 & 90 & 80 & 75 \\
R2 (8 elements) & 100 & 100 & 100 & 100 \\
R3 (9 elements) & 78 & 85.7 & 85.7 & 83 \\
\hline
\end{tabular}

commonly identified elements. Thirdly, we listed all identified connections between the common elements and checked for agreement. Lastly, the type of connection was checked for agreement. Overall, all percentages of agreement are high (> 75\%), which indicates that two independent authors made very similar DBDs from the same data based on the methodology as it is described in this paper.

\section{Results}

In the following subsections, we discuss the selected data using the dynamic blending analysis. Each DBDs is built starting from the respective transcript and visualizes the reasoning of the students. In the discussion of each DBDs, we focus on the role of graphs in the reasoning. The students' names were replaced by pseudonyms to guarantee anonymity.

\section{Case 1: Brooke and Caitlyn - Interview Task 1}

In the DBDs in Fig. 5, we present the reasoning of Brooke and Caitlyn when answering interview task 1 (Fig. 1). In their answer to subquestion A, they immediately link the formulation "no heat can flow in or out of the rod" to "there is no change there, so the derivative at the sides should be zero" (elements 1, 2, and 3). Remark that the students do not mention whether the change and partial derivative are to be understood with respect to $t$ or with respect to $x$.

Based on the physical argument that heat cannot leave an isolated system (elements 4 and 6) and should spread out evenly (element 5), in response to subquestion B, the students sketch the graphs in element 7. After drawing the graphs, the students remark that the temperature does change over time at the sides (element 8). This is unexpected for the students, and, in retrospect, it shows that the (lack of) change in element 2 is to be understood as a change with respect to time, as is the partial derivative in element 3 (which are therefore colored black). In element 9, the students state that the time derivative changes. Based on the context of this statement, we think that the students use the wrong wording and actually mean that the time derivative is non-zero at the sides, i.e., a reformulation of element 8 in terms of a partial derivative. Constructing the graphs helped the students to come to the insights in elements 8 and 9: the temperature changes over time at the sides, so the 


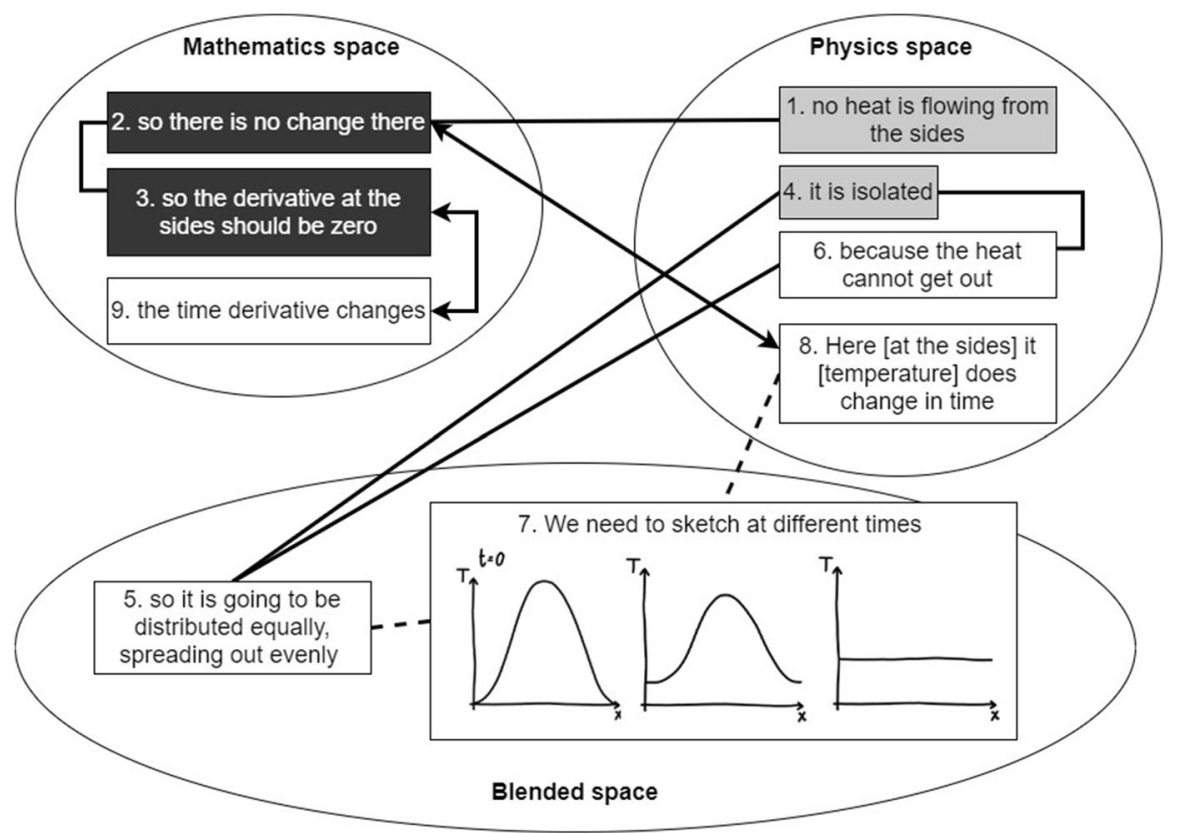

Fig. 5 DBDs of case 1, the reasoning of Brooke and Caitlyn in response to interview task 1 (Fig. 1)

partial derivative with respect to time is not zero, which contradict with their earlier ideas in elements 2 and 3. We see that students blend physical concepts with mathematical concepts.

Remark that the graphs in element 7 have horizontal tangents at the boundaries, which is in accordance with $\partial u / \partial x=0$ (the correct answer to the question), but we have no elements in the transcript that confirm that the students explicitly made this connection.

\section{Case 2: Brooke and Caitlyn - Interview Task 2C (Parts II and III)}

The second case is about the same students answering task 2C. Before the episode represented in the DBD in Fig. 6, Brooke and Caitlyn did not use the partial derivative $\partial u / \partial x$. In their earlier answers (tasks $1,2 \mathrm{~A}$ and 2B) they incorrectly used $\partial u / \partial t$ to discuss heat flow. Triggered by the $\partial u / \partial x$ in the problem statement of task $2 \mathrm{C}$ (Figs. 2 and 3), they start to doubt: "But what is heat flow then? A derivative with respect to position or to time?" After some discussion, they get to a point where they contrast both partial derivatives, but fail to distinguish clearly between the meanings of both. They show an understanding of $\partial u / \partial t$ as being the change of temperature over time, but there is an indication that the time dimension is also important in their interpretation of $\partial u / \partial x=0$ : "the temperature has to stay constant locally." Although "locally" refers to the spatial dimension, "stay constant" could be interpreted as referring to the time dimension. 


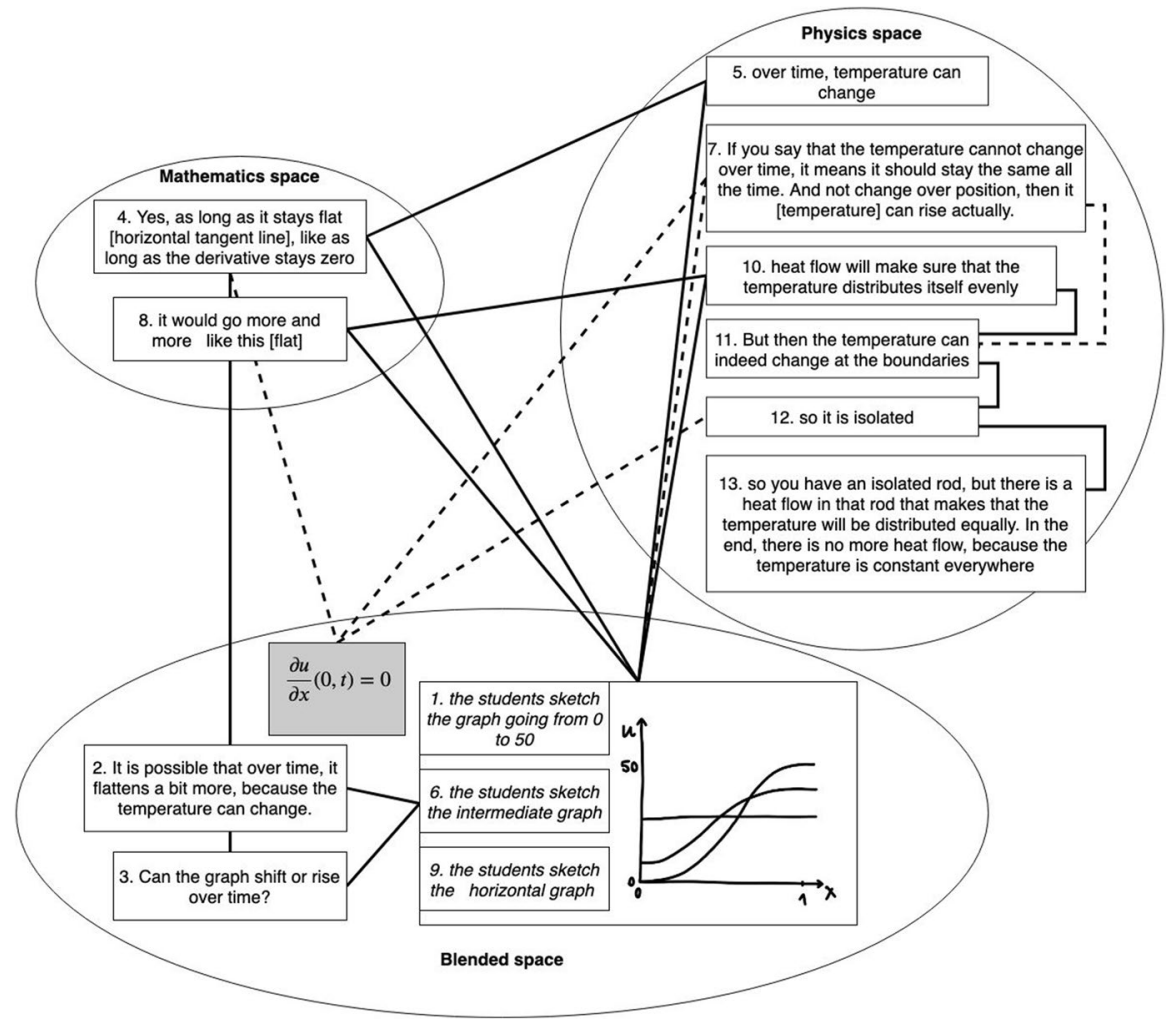

Fig. 6 DBDs of case 2, the reasoning of Brooke and Caitlyn in response to interview task 2C (Figs. 2 and 3)

From this point on, graphical reasoning comes in. Here, we start to visualize the reasoning in a DBD (see Fig. 6). In trying to understand the physical meaning of the boundary conditions, the students first sketch a graph of a hypothetical initial situation that agrees with these boundary conditions (element 1 ), then make a sketch of the temperature distribution after some time (element 6) and finally make a sketch of the limiting temperature distribution (element 9). The horizontal tangents at the boundaries in the graphs are now explicitly connected with the derivative (element 4), which shows that the students have a correct graphical interpretation of the partial derivative with respect to $x$ being zero. The graphs trigger them to formulate the difference between the two partial derivatives in physical terms (elements 5 and 7), and, therefore, we consider this as a blended element. However, again, they do this in terms of temperature change over time. Stated in terms of partial derivatives, element 7 could be interpreted as follows: $\partial u / \partial t=0$, meaning that temperature cannot change over time; $\partial u / \partial x=0$, meaning that temperature can change over time. If we compare this to the DBD of task 1 (Fig. 5), which also represents a discussion on an isolated system, we see that these students already knew that in an isolated system, the temperature can 
change over time at the boundaries. In their answer to this task, however, they add the insight that an isolated system is described by boundary conditions of the form $\partial u / \partial x=0$. This implies that their understanding improved over the course of the interview.

Element 10 shows how the students think about the evolution of the system in physical terms using the concept of heat flow. The connected elements 8 and 9 and elements 2 and 6 show that they are able to translate this evolution correctly in mathematical and graphical terms: successive graphs "flattening out," with a horizontal line as limiting case. They relate zero and non-zero heat flow in the system to graphs expressing constant or non-constant temperature profiles, respectively (element 13). We conclude that the graphs acted like a catalyst in the reasoning, guiding the students to new insights (elements 6-7, elements 9-10), but that the graphs did not lead to a complete understanding: even though the students identify $\partial u / \partial x=0$ at the boundaries as describing an isolated system (element 12), we did not see any proof that these students see $\partial u / \partial x$ as representing heat flow.

\section{Case 3: David and Evan - Interview Task 1 (Parts B and C)}

The third case presents the reasoning of a new student pair, David and Evan, in answer to interview task 1 (Fig. 1). The DBDs starts when the students answer subquestion $\mathrm{B}$, because this is where graphical reasoning starts playing a role (see

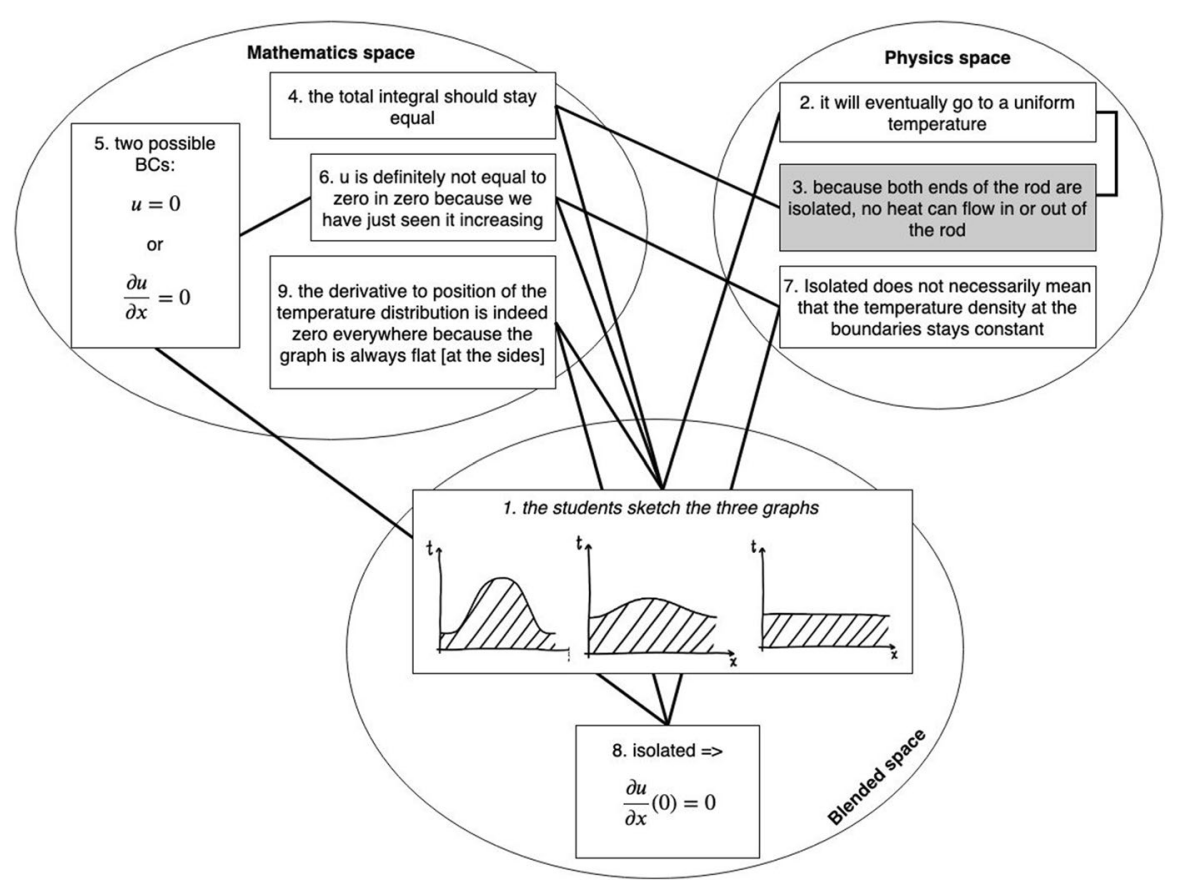

Fig. 7 DBD of case 3, the reasoning of David and Evan in response to interview task 1 (see Fig. 1) 
Fig. 7). The students begin by sketching the evolution of the temperature distribution in three graphs (element 1) blending physical (elements 2 and 3) and mathematical reasons (element 4) that no heat can flow out of the system and that therefore the surface under the graphs should stay constant. The horizontal tangent lines seem to be a coincidental attribute at this point, as the students have not consciously paid attention to this when graphing. From these sketches, they start looking for a way to describe these boundary conditions mathematically. They recall two possible options, probably from class $u=0$ and $\partial u / \partial x=0$ (element 5). From the graphs, they see clearly that the temperature does not stay zero at the sides, so they eliminate the option $u=0$ (elements 6 and 7). Therefore, they state that isolated should mean $\partial u / \partial x=0$ (element 8). Only then, they see that this agrees with their graphs: the slope with respect to $x$ is always zero on the sides (element 9). This confirms their choice.

\section{Case 4: David and Evan - Interview Task 2D}

In the fourth case, we look at the answer of the same students to task 2D (Figs. 2 and 3). With the mixed boundary conditions $\partial u / \partial x(0, t)=-2$ and $u(1, t)=4$, task $2 \mathrm{D}$ is the most challenging one in the interview. It can be interpreted in physical terms as one side having a constant, non-zero heat flow, and the other side being in contact with a heat reservoir at constant temperature. We visualize the reasoning in the DBDs in Fig. 8. The students start by interpreting the boundary condition at position zero in a physical way: one end constantly "loses heat" (element 1). This idea of losing heat might be associated with an incorrect physical interpretation of the minus sign. From elements 2 and 3, we infer that these students associate outflowing heat with a decrease in temperature, which is not necessarily true. In trying to interpret the boundary condition $\partial u / \partial x(0, t)=-2$ physically, they compare it with what they have found in earlier tasks: $\partial u / \partial x(0, t)=0$ means perfect thermal isolation (elements 5 and 6 ). However, they express to not fully understand this connection ("I find that weird"). They interpret $u(1, t)=4$ without any problem as the side being in contact with a heat reservoir (not in diagram). Separately, they also take a mathematical approach in interpreting the boundary conditions: a line with a constant, negative slope (element 4 ) and a set value of four where the slope does not matter (element 7), and they sketch this in a set of axes (element 9).

At this point, the students state that they see two possible extremes in how the system evolves graphically (scenarios 1 and 2 in elements 10 and 14, respectively). In the first scenario (10), the area under the graph decreases (11). However, they instantly have several objections against this scenario. In element 12 , they state that the heat reservoir at position 1 will provide heat to flow in. Here, students use the graphs for their mathematical (11) and physical (12) interpretations. Remarkably, in element 13 , they add that at the left boundary, "there should always be a coefficient of minus two flowing in." Here, it seems that the students imply that $\partial u / \partial x(0, t)=-2$ indicates an inward heat flow, which contradicts with their original statement in element 1 . As it is unclear from the transcript if the students notice that they said the reverse in element 1 , we do not add double arrow lines. The graphs 


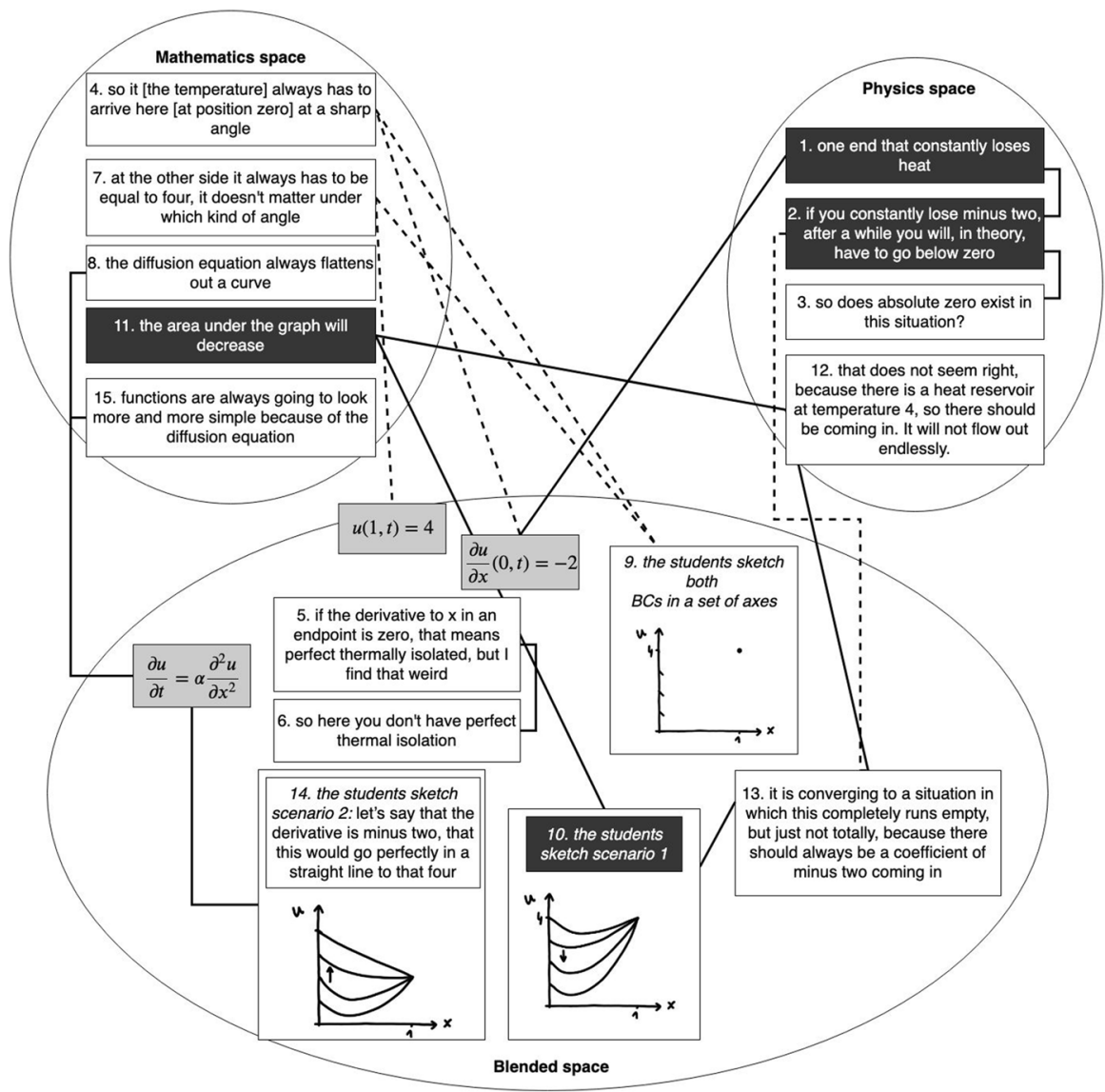

Fig. 8 DBDs of case 4, the reasoning of David and Evan in response to interview task 2D (see Figs. 2 and 3)

might have played a role in this change of mind. However, we cannot know this from the data. Overall, however, the graphs play a big role in this part of the reasoning: they trigger the arguments in elements 11,12 , and 13 , which result in rejecting scenario 1 . Next, the students sketch the second scenario: the graph converges to a straight line with a slope of -2 passing through point $(1,4)$ (element 14$)$. They immediately choose this scenario because of their earlier idea in element 8 , which they repeat and rephrase in 15: graphs should become more simple and flat over time because of the diffusion equation.

Generally, from the interpretation of the boundary condition at position zero in element 1 , we can assume that the students connect $\partial u / \partial x$ to heat flow. They also make the connection in the special case where $\partial u / \partial x=0$ and the system is isolated (elements 5 and 6). However, they mention that they do not understand where this connection is coming from. We do not see proof that they can interpret $\partial u / \partial x$ as expressing a temperature difference between two positions, which 
causes heat to flow in a direction that depends on that temperature difference. We would expect that this would play a role in deciding between scenarios 1 and 2 , but in this case, it did not. These students arrive at the correct conclusion about the equilibrium distribution, which is graphically represented by a decreasing straight line. However, their choice for the second scenario seems to be based on rather vague arguments (elements 8 and 15, the diffusion equation leading to a simple function). They do not reason in terms of heat entering at the left, flowing through the system and flowing out of the system to the heat reservoir at the right and its relation to $\partial u / \partial x$ (if this derivative is constant in the entire system, the $u(x)$ graph must be a straight line).

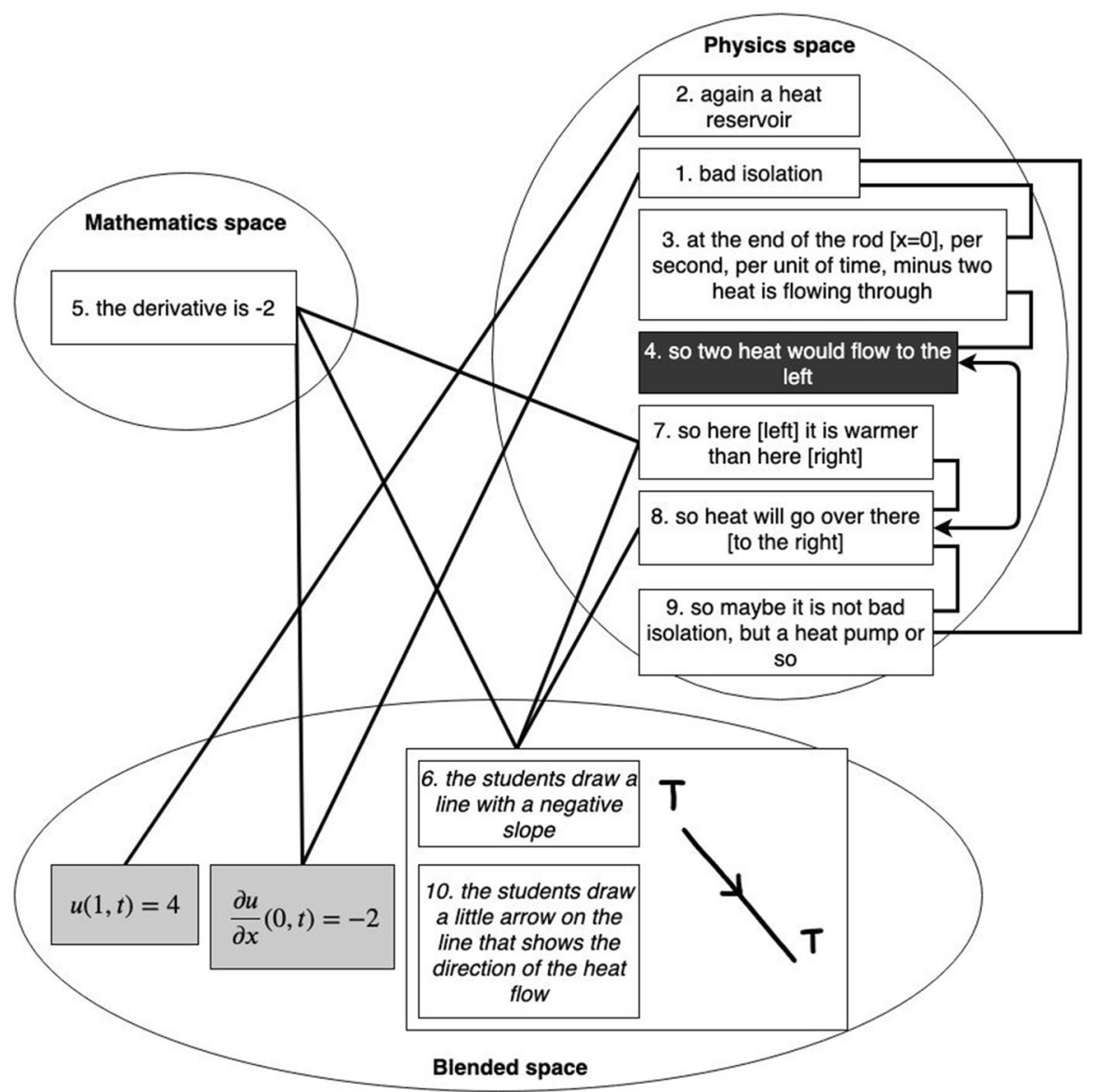

Fig. 9 DBDs of case 5, the reasoning of Fin and Glen in response to interview task 2D (see Figs. 2 and 3) 


\section{Case 5: Fin and Glen - Interview Task 2D}

In the DBD in Fig. 9, we visualize the reasoning of a third pair of students, Fin and Glen, in answer to the same interview task 2D (Figs. 2 and 3). We selected this part because it shows reasoning based on temperature differences, which was missing in the reasoning from David and Evan to task 2D shown above.

In answer to subquestion I, elements 1 and 2 show that the students use their answers to previous tasks, where they have reasoned with heat reservoirs and isolated systems. In their initial physical approach, they think that heat is flowing out of the rod at position 0 (elements 3 and 4), which is similar to the initial response of other pairs of students (e.g., case 4). The interviewer asks for further explanation, which triggers them to sketch what that boundary condition looks like graphically: a decreasing line (element 6). They come to mathematical insight by making the implicit connection between derivative and slope. In element 7, they state that the left part of the rod is warmer than the right part, which means that they attach physical meaning to the sketch. Implicitly, the students use that heat flows from positions with higher temperature to positions with lower temperature to conclude that heat will flow to the right at position zero (elements 8 and 10). The students notice the inconsistency in their reasoning (visualized by the double arrow line between element 4 and 8) and reject their initial thought (element 4) based on this graphical argument. This is an example of how graphical reasoning can help students in constructing new insights and correct their own intuitive thinking.

When continuing reasoning (not captured in the DBD), the students arrive at the correct sketch of the temperature in the physical system after a long time (a straight line given by $u(x)=-2 x+6$ ). However, when asked to explain why this is the limit, they state that they do not know the physical meaning of this limit. This shows that even though we have clear evidence that the students have good understanding of how temperature differences lead to heat flow and how this is described using $\partial u / \partial x$, these students apparently still have problems to use this knowledge in formulating an answer to the question.

\section{Discussion and Implications}

In this study, we investigated how graphs and reasoning based on those graphs help students in connecting the physical and mathematical meaning of the partial derivatives $\partial u / \partial x$ and $\partial u / \partial t$. We selected five cases from paired interviews with undergraduate students where we provided them with tasks about boundary conditions for one-dimensional systems modeled by the heat equation. Although not explicitly asked in each of the questions, being able to physically interpret the partial derivatives $\partial u / \partial x$ and $\partial u / \partial t$ allows for a more complete answer to the tasks. As mentioned before, the interview tasks were not developed to explicitly elicit graphical reasoning, but rather to focus on blending mathematical and physical knowledge. However, during the interviews and in the transcripts, we remarked the positive effect of graphs and decided to investigate it in more detail. We analyzed the data from a 
dynamic blending perspective and focused on the role of graph construction in student reasoning.

First, we observed that constructing graphs sometimes helped students to eliminate options to formulate the boundary conditions in mathematical terms. In case 1 , Brooke and Caitlyn inferred from their graphs that $\partial u / \partial t=0$ is not a suitable description for what happens at the borders of an isolated system. However, the graph did not trigger them to look at the derivative with respect to position. In case 3, we observed a similar process: the graph helped the students to realize that $u\left(x_{b}, t\right)=0$ is not a suitable boundary condition, upon which they eliminated this, and chose the correct mathematical description $\partial u / \partial x\left(x_{b}, t\right)=0$. They did not use the graph to arrive at this conclusion, but after having found out by elimination that $\partial u / \partial x\left(x_{b}, t\right)=0$, they realized that the graph they constructed earlier complied with this condition.

Second, we observed that graphs have the potential to build the connection between $\partial u / \partial x$ and heat flow. Most students used their constructed graphs to reason about this relationship, although some (case 5) more detailed than others (cases 2 and 4). In cases 2 and 4, the students made a superficial connection between $\partial u / \partial x$ and heat flow. They mentioned $\partial u / \partial x$ as representing heat flow, but it is unclear whether they know where this relationship comes from. Both pairs used the $u(x)$ graphs and refer to the slope in the $x$ direction and, as such, show to understand the graphical meaning of the partial derivative. However, they did not connect the derivative, being the limit of a difference quotient, to a temperature difference between different positions. Instead, they immediately jumped to heat flow without reasoning. This shows that the graphical interpretation of the partial derivative as slope appears helpful to build the connection, but without a good insight in the process underlying the concept of partial derivative (Zandieh, 2000), students will not be able to connect the mathematical and the physical meaning. In case 5, the graphical aspect played a central role in connecting the mathematical ideas of a (partial) derivative and slope to the physical ideas of temperature differences and heat flow. As observed in the other cases, the students started by interpreting $\partial u / \partial x$ as the slope of the $u(x)$ graph. What makes this case different is the explicit physical interpretation of the graph with $u$ being the temperature at a certain position $x$. From this, the students inferred that a negative slope means that there is a temperature difference between neighboring points. This brings them back to the physics space, where they explain that heat flows from positions with high temperature to positions with lower temperature.

Third, the graphs showed to be promising to help students to give a physical meaning to the sign of the partial derivative, being related to the direction in which heat flows. This is an aspect of the relation between $\partial u / \partial x$ and heat flow discussed in the previous paragraph, but it deserves some more attention. In task 2D, we elicited the focus on direction using the boundary condition $\partial u / \partial x(0, t)=-2$. All pairs (David and Evan in case 4, Fin and Glen in case 5, but also the ones not discussed here) initially interpreted the minus sign as referring to an outward direction of heat flow, which is not true in this case. In case 5, we observed how graphing helped the students in understanding how the sign of the slope with respect to $x$ gives information about the direction of the heat flow. Again, the necessary steps are to interpret 
the partial derivative as a limit of a difference quotient, which expresses a temperature difference between neighboring points in this physical context. Combining this with the definition of heat, which flows from points at high to points at low temperature, gives the desired insight. In case 4, we observed that the students changed their opinion about the direction of the heat flow without much explanation. It is possible that the idea of temperature differences played a role in this process, but there is no evidence that it was made explicit. From literature, we know that novices struggle to understand the many roles a negative sign can play in physics contexts (Brahmia et al., 2020; Huynh \& Sayre, 2019). Recently, Brahmia et al. (2020) developed a framework for categorizing the natures of negativity in introductory physics (NoNIP) that sheds light on this misinterpretation. In terms of NoNIP, we hypothesize that the initially incorrect interpretation of the minus sign in this study is an incorrect categorization of the physical phenomenon described by the negative partial derivative. The students probably interpret the negative partial derivative as energy that is subtracted from the system. However, it actually relates to the temperature difference between neighboring points that determines the direction of the heat flow.

To conclude, the results in this paper indicate that stimulating graphical reasoning is a promising way to support the blending of mathematical and physical knowledge. Specifically, our data showed that constructing graphs can be a catalyst that helps students to give physical meaning to the partial derivatives $\partial u / \partial t$ and/or $\partial u / \partial x$. However, even though constructing graphs helped students to progress in their reasoning, in most cases, it was not sufficient to arrive at a complete understanding of the mathematical and physical aspects of the task. Therefore, in teaching/learning activities, actively promoting graph construction and reasoning based on those graphs offers perspectives to support the blending of mathematics and physics. However, more scaffolding towards linking central physical concepts to their mathematical description and the underlying mathematical constructions is necessary. In the context of the heat equation, to teach the relation between $\partial u / \partial x$ and heat flow, the instructor should provide scaffolding towards the underlying line of reasoning starting from the partial derivative with respect to position, connecting this to a difference quotient, which can be physically interpreted by relating it to a temperature difference, and which finally leads to heat flow. The results of this paper show that graphs have the potential to play a key role in making the connection between the mathematical and the physical knowledge necessary to understand this sequence of concepts.

\section{Limitations}

As we were interested in obtaining deep insight in student reasoning, we used openended, task-based interviews with the context of the heat equation as an example. Although the interviews gave us rich data and insights into students' reasoning, it was not always possible to have a complete picture of students' reasoning and some interpretations may be ambiguous. Many factors play a role in this, like the formulation of the tasks, the role of the interviewer, and the fact that interviews only give a 
partial display of the reasoning process. By giving detailed representations of the reasoning processes of the student pairs, we show how we interpreted students' utterances. This kind of interpretative analysis may be subject to personal interpretation by the researcher. By repeated analysis by multiple researchers, however, we tried to guarantee the reliability of our analysis.

Acknowledgements We want to thank all participating students for their voluntarily participation to our interviews.

\section{References}

Arcavi, A. (2003). The role of visual representations in the learning of mathematics. Educational Studies in Mathematics, 52(3), 215-241.

Arnon, I., Cottrill, J., Dubinsky, E., Oktaç, A., Roa Fuentes, S., Trigueros, M., \& Weller, K. (2014). APOS Theory. Springer. https://doi.org/10.1007/978-1-4614-7966-6

Bain, K., Rodriguez, J.-M.G., Moon, A., \& Towns, M. H. (2018). The characterization of cognitive processes involved in chemical kinetics using a blended processing framework. Chemistry Education Research and Practice, 19(2), 617-628. https://doi.org/10.1039/C7RP00230K

Becker, N., \& Towns, M. (2012). Students' understanding of mathematical expressions in physical chemistry contexts: An analysis using Sherin's symbolic forms. Chemistry Education Research and Practice, 13(3), 209-220. https://doi.org/10.1039/C2RP00003B

Beichner, R. J. (1994). Testing student interpretation of kinematics graphs. American Journal of Physics, 62(8), 750-762.

Bing, T. J., \& Redish, E. F. (2007). The Cognitive Blending of Mathematics and Physics Knowledge, 883, 26-29.

Bollen, L., van Kampen, P., Baily, C., \& De Cock, M. (2016). Qualitative investigation into students' use of divergence and curl in electromagnetism. Physical Review Physics Education Research, 12(2), 020134.

Brahmia, S., Olsho, A., Smith, T. I., \& Boudreaux, A. (2020). Framework for the natures of negativity in introductory physics. Physical Review Physics Education Research, 16(1), 010120. https://doi.org/ 10.1103/PhysRevPhysEducRes.16.010120

Clough, E. E., \& Driver, R. (1986). A study of consistency in the use of students' conceptual frameworks across different task contexts. Science Education, 70(4), 473-496. https://doi.org/10.1002/sce.37307 00412

Eichenlaub, M., \& Redish, E. F. (2019). Blending physical knowledge with mathematical form in physics problem solving. In G. Pospiech, M. Michelini, \& B.-S. Eylon (Eds.), Mathematics in Physics Education (pp. 127-151). Springer International Publishing. https://doi.org/10.1007/ 978-3-030-04627-9_6

Farlow, S. J. (1993). Partial differential equations for scientists and engineers. Courier Corporation.

Fauconnier, G., \& Turner, M. (1998). Conceptual integration networks. Cognitive Science, 22(2), $133-187$.

Fauconnier, G., \& Turner, M. (2003). The way we think: Conceptual blending and the mind's hidden complexities. Basic Books.

Gerson, H., \& Walter, J. (2008). How blending illuminates understandings of calculus. In Electronic proceedings for the eleventh special interest group of the mathematical association of America on research in undergraduate mathematics.

Glazer, N. (2011). Challenges with graph interpretation: A review of the literature. Studies in Science Education, 47(2), 183-210. https://doi.org/10.1080/03057267.2011.605307

Goedhart, M. J., \& Kaper, W. (2003). From chemical energetics to chemical thermodynamics. In J. K. Gilbert, O. De Jong, R. Justi, D. F. Treagust, \& J. H. Van Driel (Eds.), Chemical Education: Towards Research-based Practice (pp. 339-362). Springer Netherlands. https://doi.org/10.1007/0306-47977-X_15 
Gregorcic, B., \& Haglund, J. (2021). Conceptual blending as an interpretive lens for student engagement with technology: Exploring celestial motion on an interactive whiteboard. Research in Science Education, 51, 235-275. https://doi.org/10.1007/s11165-018-9794-8

Haciomeroglu, E. S., Aspinwall, L., \& Presmeg, N. C. (2010). Contrasting cases of calculus students' understanding of derivative graphs. Mathematical Thinking and Learning, 12(2), 152-176.

Hu, D., \& Rebello, N. S. (2013). Using conceptual blending to describe how students use mathematical integrals in physics. Physical Review Special Topics-Physics Education Research, 9(2), 020118.

Huynh, T., \& Sayre, E. C. (2019). Blending of conceptual physics and mathematical signs. ArXiv:1909.11618 [Physics]. http://arxiv.org/abs/1909.11618

Johansson, H. (2016). Mathematical reasoning requirements in Swedish national physics tests. International Journal of Science and Mathematics Education, 14(6), 1133-1152. https://doi.org/10. 1007/s10763-015-9636-3

Kaiser, G., Blum, W., Ferri, R. B., \& Stillman, G. (2011). Trends in teaching and learning of mathematical modelling: ICTMA14 (Vol. 1). Springer Science \& Business Media.

Karam, R. (2015). Introduction of the thematic issue on the interplay of physics and mathematics. Science \& Education, 24(5-6), 487-494.

Kesidou, S., \& Duit, R. (1993). Students' conceptions of the second law of thermodynamics-An interpretive study. Journal of Research in Science Teaching, 30(1), 85-106.

Leinhardt, G., Zaslavsky, O., \& Stein, M. K. (1990). Functions, graphs, and graphing: Tasks, learning, and teaching. Review of Educational Research, 60(1), 1-64. https://doi.org/10.3102/0034654306 0001001

Linn, M. C., \& Songer, N. B. (1991). Teaching thermodynamics to middle school students: What are appropriate cognitive demands? Journal of Research in Science Teaching, 28(10), 885-918. https://doi.org/10.1002/tea.3660281003

McDermott, L. C., Rosenquist, M. L., \& Van Zee, E. H. (1987). Student difficulties in connecting graphs and physics: Examples from kinematics. American Journal of Physics, 55(6), 503-513.

Niss, M. (2017). Obstacles related to structuring for mathematization encountered by students when solving physics problems. International Journal of Science and Mathematics Education, 15(8), 1441-1462. https://doi.org/10.1007/s10763-016-9754-6

Planinic, M., Milin-Sipus, Z., Katic, H., Susac, A., \& Ivanjek, L. (2012). Comparison of student understanding of line graph slope in physics and mathematics. International Journal of Science and Mathematics Education, 10(6), 1393-1414. https://doi.org/10.1007/s10763-012-9344-1

Pospiech, G., Michelini, M., \& Eylon, B.-S. (Eds.). (2019). Mathematics in physics education. Springer International Publishing. https://doi.org/10.1007/978-3-030-04627-9

Redish, E. F., \& Kuo, E. (2015). Language of physics, language of math: Disciplinary culture and dynamic epistemology. Science \& Education, 24(5-6), 561-590.

Rodriguez, J.-M.G., Bain, K., \& Towns, M. H. (2020). Graphical forms: The adaptation of Sherin's symbolic forms for the analysis of graphical reasoning across disciplines. International Journal of Science and Mathematics Education, 18(8), 1547-1563. https://doi.org/10.1007/ s10763-019-10025-0

Rodriguez, J.-M.G., Bain, K., Towns, M. H., Elmgren, M., \& Ho, F. M. (2019). Covariational reasoning and mathematical narratives: Investigating students' understanding of graphs in chemical kinetics. Chemistry Education Research and Practice, 20(1), 107-119. https://doi.org/10.1039/ C8RP00156A

Rodriguez, J.-M.G., Santos-Diaz, S., Bain, K., \& Towns, M. H. (2018). Using symbolic and graphical forms to analyze students' mathematical reasoning in chemical kinetics. Journal of Chemical Education, 95(12), 2114-2125. https://doi.org/10.1021/acs.jchemed.8b00584

Roth, W.-M., \& Bowen, G. M. (2003). When are graphs worth ten thousand words? An Expert-Expert Study. Cognition and Instruction, 21(4), 429-473.

Roundy, D., Bridget Kustusch, M., \& Manogue, C. (2014). Name the experiment! Interpreting thermodynamic derivatives as thought experiments. American Journal of Physics, 82(1), 39-46. https://doi. org/10.1119/1.4824548

Schermerhorn, B. (2018). Investigating student understanding of vector calculus in upper-division electricity and magnetism: Construction and determination of differential element in non-cartesian coordinate systems [University of Maine]. https://digitalcommons.library.umaine.edu/etd/2844 
Schermerhorn, B. P., \& Thompson, J. R. (2019). Physics students' construction of differential length vectors in an unconventional spherical coordinate system. Physical Review Physics Education Research, 15(1), 010111. https://doi.org/10.1103/PhysRevPhysEducRes.15.010111

Shah, P., Mayer, R. E., \& Hegarty, M. (1999). Graphs as aids to knowledge construction: Signaling techniques for guiding the process of graph comprehension. Journal of Educational Psychology, 91(4), 690-702.

Sherin, B. L. (2001). How students understand physics equations. Cognition and Instruction, 19(4), 479-541.

Thompson, J. R., Bucy, B. R., \& Mountcastle, D. B. (2006). Assessing student understanding of partial derivatives in thermodynamics. AIP Conference Proceedings, 818(1), 77-80. https://doi.org/10. $1063 / 1.2177027$

Thompson, J. R., Manogue, C. A., Roundy, D. J., \& Mountcastle, D. B. (2012). Representations of partial derivatives in thermodynamics. AIP Conference Proceedings, 1413, 85-88.

Uhden, O., Karam, R., Pietrocola, M., \& Pospiech, G. (2012). Modelling mathematical reasoning in physics education. Science \& Education, 21(4), 485-506.

Wilcox, B. R., Caballero, M. D., Rehn, D. A., \& Pollock, S. J. (2013). Analytic framework for students' use of mathematics in upper-division physics. Physical Review Special Topics-Physics Education Research, 9(2), 020119.

Zandieh, M. (2000). A theoretical framework for analyzing student understanding of the concept of derivative. CBMS Issues in Mathematics Education, 8, 103-127.

Zandieh, M., Roh, K. H., \& Knapp, J. (2014). Conceptual blending: Student reasoning when proving "conditional implies conditional" statements. Journal of Mathematical Behavior, 33(C), 209-229. https://doi.org/10.1016/j.jmathb.2013.11.007

Zavala, G., Tejeda, S., Barniol, P., \& Beichner, R. J. (2017). Modifying the test of understanding graphs in kinematics. Physical Review Physics Education Research, 13(2), 020111.

Zazkis, D. (2013). Prompted and unprompted transitions between representational modes in calculus. In M. Martinez \& A. Castro Superfne (Eds.), Proceedings of the 35th annual meeting of the North American Chapter of theInternational Group for the Psychology of Mathematics Education (pp. 1232-1239). University of Illinois at Chicago. 